\title{
Follow-up of multiple sclerosis patients treated with Endotherapia (GEMSP)
}

\author{
MICHEL GEFFARD $^{1}$, ARTURO MANGAS ${ }^{2}$ and RAFAEL COVEÑAS ${ }^{3}$ \\ ${ }^{1}$ Institut pour le Développement de la Recherche en Pathologie Humaine et Thérapeutique, 33400 Talence;
${ }^{2}$ Gemacbio, Research Department, Lieu dit Berganton, 33127 Saint Jean d'Illac, France; ${ }^{3}$ Institute of Neurosciences of
Castilla y León, Laboratory of Neuroanatomy of the Peptidergic Systems, University of Salamanca, 37007 Salamanca, Spain
}

Received November 21, 2016; Accepted January 23, 2017

DOI: $10.3892 /$ br.2017.857

\begin{abstract}
Endotherapia (GEMSP) is a novel therapeutic approach for multiple sclerosis (MS). The aim of the present study was to demonstrate the efficiency of Endotherapia in the follow-up of 193 patients with MS. The efficiency coefficient that was evaluated was the Expanded Disability Status Scale (EDSS) score, which is a functional scale of MS progression. The evaluated score of each patient during follow-up visits was compared with the theoretical score of the disease progression without GEMSP. The evolution of the EDSS score was evaluated according to the inclusion score. The quantitative global study of the EDSS score highlighted a statistically significant difference between the final average scores of the treatment with GEMSP (M) and worldwide reference (R) groups. The improvement of the $\mathrm{M}$ group compared with the $\mathrm{R}$ group was $24.5 \%$. According to the final EDSS scores, the study highlighted a difference in favor of the $\mathrm{M}$ group with $62.0 \%$ for scores $\leq 3,7.8 \%$ for scores between 3 and 6 and $19.6 \%$ for scores $\geq 6$. According to the qualitative evolution of the EDSS scores, the improvements in favor of group $\mathrm{M}$ were $49.3 \%$ for scores $\leq 3,79.1 \%$ for scores between 3 and 6 and $19.5 \%$ for scores $\geq 6$. The qualitative study of the EDSS score showed a statistically significant success percentage; the success percentages were between 59.1 and $90.0 \%$. In a larger population of MS patients, the data confirm the beneficial effects of GEMSP that were previously reported in pre-clinical and clinical studies. In addition, $78 \%$ of patients showed an improvement or deceleration of the disease.
\end{abstract}

Correspondence to: Dr Michel Geffard, Institut pour le Développement de la Recherche en Pathologie Humaine et Thérapeutique, 200 Avenue de Thouars, 33400 Talence, France E-mail: mangasam@usal.es; mg.idrpht@orange.fr

Key words: multiple sclerosis, Expanded Disability Status Scale score, endotherapia, GEMSP, poly-L-lysine

\section{Introduction}

Multiple sclerosis (MS) is a chronic, inflammatory, demyelinating and neurodegenerative disease that predominantly affects people aged between 20 and 40 years (1). The damage appears to be due to inflammatory processes in which lymphocytes become activated at the periphery, disrupt the intracellular matrix of the blood-brain barrier and invade the central nervous system. Environmental (including toxic substances, metabolic stress and possible pathogen infection) and genetic factors may facilitate the movement of auto-reactive $\mathrm{T}$ cells and demyelinating antibodies into the central nervous system (1-4).

To date, MS has no cure and only partially efficient therapeutic strategies are available [for example beta interferons, glatiramer acetate (Copaxone), Tysabri, fingolimod and laquinimod]. None of these therapeutic agents reduce the disability experienced by patients suffering from MS. Thus, novel therapeutic strategies and agents are required for the treatment of MS (5). One such therapeutic strategy/agent could be Endotherapia (GEMSP) (6,7). Endotherapia is a novel therapeutic approach against chronic conditions (for example neurodegenerative and auto-immune diseases) that accounts for genetic predisposition, and environmental, bacterial and immunological factors. This includes the identification of specific circulating antibodies in the serum of patients and the use of therapeutic tools, such as small compounds linked to the carrier poly-L-lysine (PLL) and the physiological actions of such compounds being known (e.g., GEMSP) $(6,7)$. PLL is edible, water-soluble and non-toxic for humans (8). Depending on the circulating antibodies directed against the antigens found during the course of MS, the chemical composition and doses of the therapeutic agent (e.g., GEMSP) can be established for each patient (9).

GEMSP was originally conceived for the treatment of the secondary progressive form of MS and it is a combination of amino acids, fatty acids, free radical scavengers and antioxidants linked to PLL $(6,7,9)$. The beneficial effects of GEMSP have been demonstrated in acute and chronic experimental autoimmune encephalomyelitis (EAE) models $(10,11)$. Subsequently, an open clinical trial stated that following treatment with GEMSP for 6 months, the Expanded Disability Status Scale (EDSS) improved in $18 \%$ of patients and remained unchanged in 55\% (1). Due to the promising results found in 
preclinical and clinical studies, another study with a larger number of MS patients ( $\mathrm{n}=102)$ was conducted (6) and again, treatment with GEMSP was beneficial for $72 \%$ of patients. The present study follows up the evolution of the disease in a larger population of MS patients $(n=193)$ that received GEMSP treatment.

\section{Materials and methods}

Population description. The present study was performed in 193 volunteer patients [152 women (78.8\%) and 41 men (21.2\%)]. The patients provided written informed consent and treatment subsequently commenced. The study was national, multicentre, non-blinded and non-randomized, and the experimental unit was the patient. The EDSS score (9) was evaluated, and treatment with GEMSP (M) and worldwide reference (R) scores were established and compared. The M score was evaluated in each patient following clinical evaluation, whereas the $\mathrm{R}$ score was the estimated theoretical international score evolution ( 0.25 points/year/patient). The period of treatment with GEMSP was from 150 to 7,709 days (21.1 years) and the median was 1,289 days (3.5 years). The following data for each patient affected by MS and treated with GEMSP were collected: Age, gender, date of diagnosis, start/termination of GEMSP treatment and the MS Assessment Questionnaire follow-up for each patient over time. In addition, the evolution of the EDSS score was tracked during the study. A plot of the evolution of the EDSS score (R) over time was conducted. The slope $(\mathrm{P}=0.25)$ corresponds to the mean $\mathrm{R}$ of the speed of EDSS evolution ( 0.25 point/year), allowing the evolution of individual scores to be compared with the worldwide mean trend of progression. Finally, the duration of the GEMSP treatment, the number of medical consultations and the interval between these consultations were also taken into consideration.

Score. The individual EDSS introduced fluctuations along time. In order to assess the total effect of treatment with GEMSP for each patient, the individual mean of EDSS evolution was determined over time as follows: i) When the individual mean rate of EDSS evolution with GEMSP was greater or equal to the mean speed of the worldwide EDSS reference, this indicated disease progression (a worsening of the state of the patient); ii) when the individual mean rate of EDSS evolution with GEMSP was recorded to be between the mean speed of the worldwide EDSS reference and 0, this indicated a decrease in the progression of the disease; iii) when the individual mean rate of EDSS evolution with GEMSP was equal to 0, this indicated a stabilization of the disease; and iv) when the individual mean rate of EDSS evolution with GEMSP was below 0, the evolution of the disease was reversed (an improvement of the disease and a recovery from lesions occurred).

Statistical analysis. In order to overcome the high variability of the MS cohort included in the current study, a method of adapted valuation was applied as previously described (6). Thus, the Mann-Whitney U test was used to compare the current population with the population of reference [where the score evolution of each patient was compared with the worldwide reference score (R)], allowing the comparison of patients with each other and to assess the efficacy of treatment with GEMSP. $\mathrm{P}<0.05$ was considered to indicate a statistically significant difference and analyses were performed using the version 9 software (SAS Institute Inc., Cary, NC, USA); the conditions of the test application were verified in order to confirm that the statistics were correctly conducted. A comprehensive study of the EDSS score and the EDSS evolution was conducted. In the descriptive analysis, the following parameters were investigated: Strength, percentage, distribution, minimum, $1^{\text {st }}$ quartile, median, $3^{\text {rd }}$ quartile, maximum (Max), average, standard deviation and $95 \%$ confidence interval. In the comparative analysis, a t-test between the $\mathrm{M}$ group and $\mathrm{R}$ group final scores was performed. Equality of the variance was verified using the Folded $\mathrm{F}$ test. In the descriptive analysis the following parameters were evaluated: Strength, percentage and distribution. Regarding the analysis of the evolution of the data by a linear regression model, the following parameters were analyzed for the $\mathrm{M}$ and $\mathrm{R}$ scores: Coefficient of correlation, $\mathrm{R}^{2}$ and the associated P-value, intercept and slope $(\mathrm{ax}+\mathrm{b})$. For comparison of the success/failure rates, the $\chi^{2}$ test or Fisher's exact test were applied according to the theoretical strength obtained by unilateral assumption (\% success $>\%$ failure).

Furthermore, a qualitative study of the EDSS score (global study) was conducted. In the descriptive analysis the following parameters were evaluated: Strength, percentage and distribution. For comparison of the success/failures rates: The $\chi^{2}$ test or Fisher's exact test were performed according to the theoretical strength obtained by unilateral assumption (\% success $>\%$ failure).

Dose and synthesis of GEMSP. GEMSP (15 mg per day) was administered via the sublingual route. GEMSP was synthesized according to patent numbers 792167 (EU) and 6114388 (USA) $(6,9)$. GEMSP is a 'tailor-made' mixture of functional polypeptides: Fatty acids linked to PLL (e.g., thioctic acid, oleic acid and linoleic acid), antioxidants linked to PLL (e.g., ascorbic acid), free radical scavengers and amino acids linked to PLL (e.g., taurine, cysteine and methionine).

\section{Results}

General considerations. Patients included in the current study were born between the $1^{\text {st }}$ September 1937 and the $1^{\text {st }}$ June 1994 (59\% of patients were born between 1951 and 1970). The diagnosis of MS was performed between the $1^{\text {st }}$ October 1962 and the $28^{\text {th }}$ April 2011 (44\% of cases were diagnosed between 1996 and 2005). The diagnosis of the disease was performed when patients were aged between 12 and 62 years (median, 36 years). The beginning of the treatment with GEMSP was between the $1^{\text {st }}$ September 1994 and the $1^{\text {st }}$ August 2011 (median, $1^{\text {st }}$ November 2006). The majority of patients began the treatment after the year 2005. The delay between the date of diagnosis and the beginning of the treatment with GEMSP was between 0 and 500 months (median, 106 months). Upon inclusion in the study, global EDSS scores were between 0 and 8.5 (median, 5); the mean score was 4.4. The median of the delay between the date of the EDSS score evaluation and the date of the treatment with GEMSP was 31 days (mean delay, 301 days). The follow-up period was between 150 and 7,709 days [21.1 years; median, 1,289 days (3.5 years)]. The number of 
Table I. Expanded Disability Status Scale global study (n=193).

\begin{tabular}{lccc}
\hline Parameter & $\begin{array}{c}\text { Score at } \\
\text { inclusion }\end{array}$ & $\begin{array}{c}\text { Final M } \\
\text { score }\end{array}$ & $\begin{array}{c}\text { Final R } \\
\text { score }\end{array}$ \\
\hline Minimum & 0.00 & 0.00 & 0.25 \\
Quartile, 25\% & 2.50 & 2.00 & 3.50 \\
Median & 5.00 & 5.50 & 5.82 \\
Quartile, 75\% & 6.50 & 6.50 & 7.23 \\
Maximum & 8.50 & 8.50 & 10.00 \\
Mean \pm SD & $4.36 \pm 2.30$ & $4.44 \pm 2.48^{\mathrm{a}}$ & $5.43 \pm 2.43$ \\
95\% CI & $4.03-4.68$ & $4.08,4.79$ & $5.08,5.77$ \\
\hline
\end{tabular}

${ }^{\mathrm{a}} \mathrm{P}<0.0001$ vs. Final R score. M, treatment with GEMSP; R, worldwide reference; $\mathrm{SD}$, standard deviation; $\mathrm{CI}$, confidence interval.

Table II. Success/failure global evolution $(n=193)$.

\begin{tabular}{lcc}
\hline Evolution & Distribution, n (\%) & Success, n (\%) \\
\hline Improvement & $51(26.4)$ & $150 / 193(77.7)^{\mathrm{a}}$ \\
Deceleration & $99(51.3)$ & \\
Degradation & $43(22.3)$ & \\
\hline
\end{tabular}

Where failure means degradation, and success is equal to the sum of deceleration and improvement. ${ }^{\mathrm{a}} \mathrm{P}<0.0001$ : Improvement + deceleration vs. degradation.

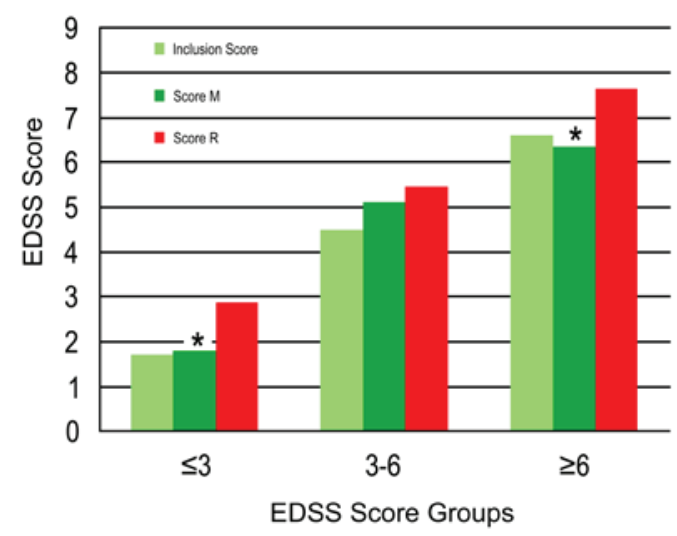

Figure 1. Comparison between the EDSS mean scores. " $\mathrm{P}<0.0001$ vs. R group. M, treatment with GEMSP; R, worldwide reference. EDSS, Expanded Disability Status Scale.

visits was between 1 and 29 (median, 7). Finally, the median interval between visits was 121 days.

EDSS global study. A statistically significant difference $(\mathrm{P}<0.0001)$ was observed between the EDSS mean scores of the $\mathrm{M}$ and $\mathrm{R}$ groups (Fig. 1 and Table I). The EDSS mean score of the M group was 4.44 and that of the $\mathrm{R}$ group was 5.43 . Regarding the EDSS mean score upon inclusion in the study, an increase of $1.8 \%$ was observed in the $\mathrm{M}$ group, whereas in
A

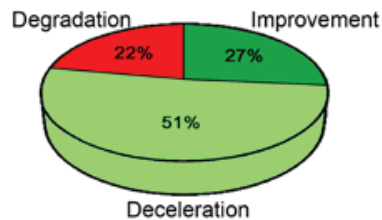

B
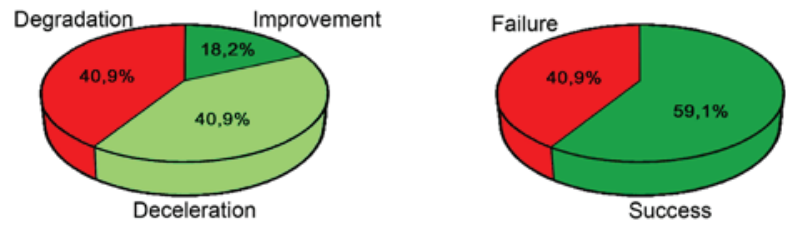

C
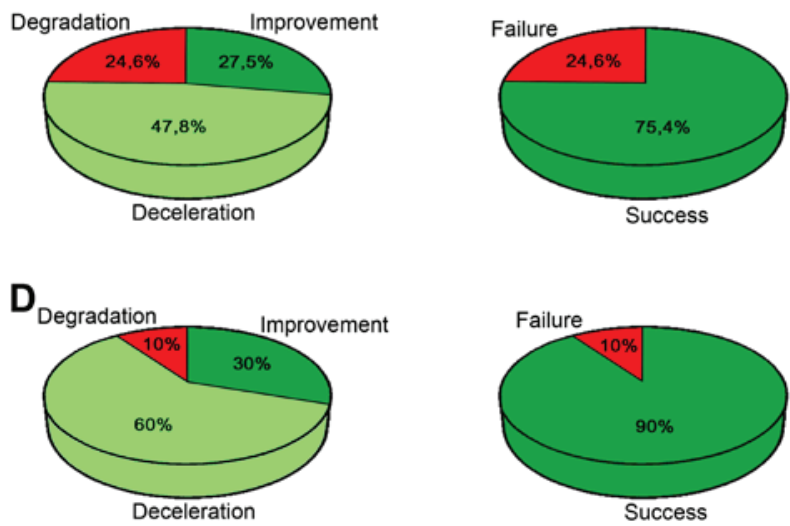

Figure 2. (A) Global evolution of patients. Evolution of patients with inclusion score (B) $\leq 3$, (C) 3-6 and (D) $\geq 6$. Where degradation refers to patients that did not respond to the therapy and evolution follows the R score. Deceleration refers to an $\mathrm{M}$ score evolution that is lower than the $\mathrm{R}$ score evolution. Improvement indicates scores of patients that were lower at the end of the study than upon inclusion. Failure means degradation, and success is equal to the sum of deceleration and improvement. M, treatment with GEMSP; R, worldwide reference.

the $\mathrm{R}$ group the increase was $24.5 \%$. In 193 patients treated with GEMSP, $22 \%$ showed a worsening of their state (degradation); $51 \%$ showed a decrease in the progression of the disease (deceleration) and $27 \%$ showed a reversal of the disease evolution, which is considered to be an improvement in their status (Fig. 2A; Table II). Thus, $78 \%$ of patients showed an improvement or deceleration of the disease (Fig. 2A). The difference between the percentages of success and failure was identified to be significantly different $(\mathrm{P} \leq 0.0001)$.

EDSS score depending on the score upon inclusion in the study. A statistically significant difference was identified between the EDSS mean scores of the $\mathrm{M}$ and $\mathrm{R}$ groups for the EDSS score $\leq 3(\mathrm{P}=0.0001)$ and $\geq 6(\mathrm{P}<0.0001)$, although this difference was not significant for the EDSS score 3-6 (Fig. 1 and Table III; $\mathrm{P}=0.20)$. According to the final EDSS scores and in comparison with the EDSS inclusion scores, the percentages of improvement for the $\mathrm{M}$ group were as follows: $62 \%$ (score $\leq 3$ ), 7.8\% (score 3-6) and 19.6\% (score $\geq 6$ ). Regarding the qualitative evolution of the EDSS scores, a statistically significant difference was identified when comparing the $\mathrm{M}$ and $\mathrm{R}$ group mean evolutions (for the scores of $\leq 3$ and $\geq 6$ ), but not in the case of score 3-6. The percentages of improvement 
Table III. Expanded Disability Status Scale score depending on the score at inclusion.

\begin{tabular}{lccc}
\hline A, Score $\leq 3$ & \multicolumn{3}{l}{} \\
\hline Parameter & Inclusion score & Final M score & Final R score \\
\hline Patients, $\mathrm{n}$ & 69 & 69 & 69 \\
Minimum & 0 & 0 & 0.25 \\
Quartile, 25\% & 1.00 & 1.00 & 1.75 \\
Median & 2.00 & 1.50 & 2.96 \\
Quartile, 75\% & 3.00 & 2.50 & 3.81 \\
Maximum & 3.00 & 7.00 & 7.53 \\
Mean \pm SD & $1.71 \pm 1.13$ & $1.80 \pm 1.47^{\mathrm{a}}$ & $2.86 \pm 1.63$ \\
95\% CI & $1.44,1.98$ & $1.45,2.16$ & $2.47,3.25$ \\
\hline
\end{tabular}

B, Score 3-6

\begin{tabular}{lccc}
\hline Parameter & Inclusion score & Final M score & Final R score \\
\hline Patients, $\mathrm{n}$ & 44 & 44 & 44 \\
Minimum & 3.50 & 1.00 & 3.66 \\
Quartile, 25\% & 4.00 & 4.50 & 4.84 \\
Median & 4.50 & 5.50 & 5.60 \\
Quartile, 75\% & 5.00 & 6.00 & 5.90 \\
Maximum & 5.50 & 7.00 & 8.52 \\
Mean \pm SD & $4.48 \pm 0.68$ & $5.11 \pm 1.49^{\mathrm{b}}$ & $5.46 \pm 0.92$ \\
95\% CI & $4.27,4.69$ & $4.66,5.57$ & $5.18,5.74$ \\
\hline
\end{tabular}

C, Score $\geq 6$

\begin{tabular}{lccc}
\hline Parameter & Inclusion score & Final M score & Final R score \\
\hline Patients, $\mathrm{n}$ & 80 & 80 & 80 \\
Minimum & 6.00 & 1.00 & 6.24 \\
Quartile, 25\% & 6.00 & 6.00 & 6.83 \\
Median & 6.50 & 6.50 & 7.50 \\
Quartile, 75\% & 7.00 & 7.00 & 8.20 \\
Maximum & 8.50 & 8.50 & 10.0 \\
Mean \pm SD & $6.58 \pm 0.59$ & $6.33 \pm 1.38$ & $7.62 \pm 0.99$ \\
95\% CI & $6.44,6.71$ & $6.02,6.64$ & $7.40,7.84$ \\
P-value & & \multicolumn{2}{c}{$<0.0001$} \\
\hline
\end{tabular}

${ }^{\mathrm{a}} \mathrm{P}<0.0001$ vs. Final R score; ${ }^{\mathrm{b}} \mathrm{P}<0.2$ vs. Final $\mathrm{R}$ score. $\mathrm{M}$, treatment with GEMSP; R, worldwide reference; SD, standard deviation; CI, confidence interval.

for the $\mathrm{M}$ group were as follows: $49.3 \%$ (score $\leq 3$ ), $79.1 \%$ (score 3-6) and 19.3\% (score $\geq 6$ ).

Evolution depending on the score upon inclusion. A statistically significant difference was observed between the percentages of success and failure for the EDSS scores 3-6 $(\mathrm{P}<0.0001)$ and $\geq 6(\mathrm{P}<0.0001)$. However, the EDSS score $\leq 3$ was at the limit of significance $(\mathrm{P}=0.088$; Table IV). In the case of those patients with an EDSS score $\leq 3$, the following
Table IV. Evolution depending on the score at inclusion.

\begin{tabular}{lccc}
\hline Score & Evolution & Distribution, n (\%) & Success, n (\%) \\
\hline$\leq 3$ & Improvement & $8(18.2)$ & $26 / 44(59.1)$ \\
& Deceleration & $18(40.9)$ & \\
& Degradation & $18(40.9)$ & \\
$3-6$ & Improvement & $19(27.5)$ & $52 / 69(75.4)^{\mathrm{a}}$ \\
& Deceleration & $33(47.8)$ & \\
\multirow{3}{*}{36} & Degradation & $17(24.6)$ & \\
& Improvement & $24(30.0)$ & $72 / 80(90.0)^{\mathrm{a}}$ \\
& Deceleration & $48(60.0)$ & \\
& Degradation & $8(10.0)$ & \\
\hline
\end{tabular}

Where failure means degradation, and success is equal to the sum of deceleration and improvement. ${ }^{\mathrm{a}} \mathrm{P}<0.0001$ : Improvement + deceleration vs. degradation.

results were observed (Fig. 2B and Table IV): Improvement (18.2\%), deceleration (40.9\%) and degradation (40.9\%); that is, success $(59.1 \%)$ and failure $(40.9 \%)$. For patients with an EDSS score 3-6, the following results were observed (Fig. 2C and Table IV): Improvement (27.5\%), deceleration (47.8\%) and degradation $(24.6 \%)$; that is, success $(75.4 \%)$ and failure (24.6\%). For patients with an EDSS score $\geq 6$, the following results were observed (Fig. 2D and Table IV): Improvement $(30 \%)$, deceleration $(60 \%)$ and degradation $(10 \%)$; that is, success $(90 \%)$ and failure $(10 \%)$. Thus, the three groups exhibited a percentage of success between 59.1 and $90.0 \%$ (Fig. 2B-D).

\section{Discussion}

Approved MS treatments are focused towards the relapsing-remitting phases, but not the progressive phases. Inflammation is important during the relapsing-remitting phase of MS, but not during the secondary progressive phase (6). The majority of the therapeutic agents currently tested for the treatment of MS act against inflammatory processes. However, existing data demonstrate that the inflammatory processes are not exclusive in the pathogenesis of MS, as there are other mechanisms involved (e.g., oxidative stress, axonal injury and neuronal loss) $(5,12)$. A global treatment for MS, including the progressive phases, is required (5). Endotherapia takes into consideration environmental, immunological and bacterial factors, and is proposed as a treatment for chronic incurable diseases exhibiting a multifactorial etiology (e.g., MS and amyotrophic lateral sclerosis). Therefore, GEMSP was developed as an anti-inflammatory therapeutic strategy (the therapeutic agent contains fatty acids), as well as for myelin/neuron protection $(1,12)$. GEMSP contains compounds (e.g., vitamins), which are effective against nitrosative and oxidative stress (1).

The linkage of heterogeneous molecules (e.g., amino acids, vitamins and fatty acids) to PLL offers various advantages as follows (6): Molecule stablility; prevention of metabolic degradation of the linked molecules; improvement of the kinetics, 
and increased half-life and permeability of membranes. Furthermore, in GEMSP, the different molecules are linked to PLL by reduced glutaraldehyde linkages resulting in particularly flexible bonds (free conformation in space improves interactions and facilitates access to the lesion site) (13). The beneficial effects of GEMSP only occur when the molecules are linked to PLL, since free constituents (not linked to PLL) are less active against MS symptoms and it seems that these free molecules either degrade or are rapidly incorporated into the metabolism (1).

In preclinical studies (acute model of EAE) (10), it has been reported that GEMSP $(0.75 \mathrm{mg} / \mathrm{day})$ inhibited brain leukocyte infiltration (10). Later, in a chronic model of EAE, it was demonstrated that GEMSP ( $7.5 \mathrm{mg} /$ day) abolished EAE episodes, the clinical score and brain leukocyte infiltration. In addition, one compound of the GEMSP (methionine) was located in the motoneurons of the spinal cord (11). This finding indicates that GEMSP may be involved in the neutralization of free radicals, exerting a neuroprotective action by inhibiting apoptotic mechanisms (11). Furthermore, in a chronic EAE model, it was demonstrated that GEMSP protected and enhanced formation of the myelin sheath (14). In a phase IIa trial, 22 patients were treated with GEMSP $(0.75 \mathrm{mg} /$ day, sublingual route) for six months. In 55\% of patients, the EDSS was stable and in $18 \%$ the EDSS decreased (instead of a normal progression of 0.25 points on the mean EDSS scale) (1). Furthermore, 28\% of patients did not react to the GEMSP treatment. No side effects (biological, hematological or hepatic) were observed and hence GEMSP was demonstrated to be safe. In addition, no toxicity was observed in experimental animals $(10 \mathrm{mg} / \mathrm{kg}$, single intravenous injection) or in humans (11). Another study was conducted where the MS patients $(\mathrm{n}=102)$ received $15 \mathrm{mg} /$ day GEMSP (sublingual route) for ranging from 3 months to 15 years and the results were as follows: $28 \%$ of patients showed a worsening of their state; $20 \%$ showed a decrease in the progression of the disease; $35 \%$ showed an improvement of their state; and $17 \%$ showed stabilization. That is, $72 \%$ of patients showed a positive evolution of the disease (6). The present study $(n=193)$ validates the favorable previous clinical study results, with 22 and 102 patients, that were previously published $(1,6)$. However, it is important to remark that the positive effect of treatment with GEMSP differs depending on the initial EDSS score of the patients. Thus, with a higher score, a greater positive effect of the treatment is observed: $59.1 \%$ of patients with initial EDSS scores $\leq 3$ showed a positive evolution; when the initial EDSS scores were between 3-6, the positive effect was found in $75.3 \%$ of patients, increasing to $90.0 \%$ when the initial EDSS scores were $\geq 6$. In the previous study performed in 102 patients (6), the percentages for the three groups (scores $\leq 3,3-6$ and $\geq 6$ ) were as follows: 74,62 and $73 \%$, respectively. In order to compare the results found in the current study $(\mathrm{n}=193)$ with those published in the previous work $(n=102)(6)$, it is important to remark that the MS patients of the latter study are included in the present work with an extended follow-up period. Regarding the group in which the initial EDSS scores at inclusion were $\leq 3$ it was found that, in the present study, the absence of efficacy of GEMSP treatment increased from 26 to $40.9 \%$ and that the improvement of the treatment decreased from 33 to $18.2 \%$ when compared with the previous study $(\mathrm{n}=102)$. The deceleration in the progression of the disability was similar in the two studies (40.9 and 41\%). Regarding the group in which the initial EDSS scores at the inclusion were 3-6, the absence of effectiveness of GEMSP treatment decreased from 38 to $24.6 \%$; deceleration in disability progression increased from 19 to $47.8 \%$ and the improvement decreased from 43 to $27.5 \%$. Regarding the group in which the initial EDSS scores at inclusion were $\geq 6$, the absence of efficacy of GEMSP treatment decreased from 26 to $10 \%$; deceleration in disability progression increased from 40 to $60 \%$ and the improvement was similar in the two studies (33 and 30\%).

Compared with other therapeutic agents, treatment with beta-interferon was demonstrated to be less efficient than treatment with GEMSP in the present study, as the former decelerates the progression of disability, whereas GEMSP improves the disability score. This finding is consistent with the results published in a previous study $(n=102)(6)$. Approved therapeutic agents for the treatment of MS exert marked side effects. Alemtuzumab is more effective than GEMSP, but the undesirable effects of alemtuzumab are important (e.g., infection, immune thrombocytopenic purpura and thyroid disorder) as are those that are caused by the use of beta-interferon (e.g., elevated liver enzymes, leukopenia, influenza-like syndrome, formation of neutralizing antibodies). However, no side effects were experienced when GEMSP was administered $(1,6,9)$. GEMSP contains compounds that are not foreign, but are endogenous; these compounds are known by the organism and, for this reason, the clinical trials performed to date have shown a good safety profile for GEMSP (neither adverse effects nor toxicity have been reported). Furthermore, other therapeutic agents, such as linomide, mitoxantrone and natalizumab are associated with considerable side effects (1). Conventional therapeutic strategies (e.g., $\beta$-interferon and glatiramer acetate) are based on immunomodulatory drugs modifying the number of immunological cells $(1,15)$, whereas GEMSP counteracts the inflammatory mechanisms, as well as other pathogenic mechanisms (e.g., oxidative stress, demyelination and neurotoxicity) (12).

In the pathogenesis of MS, oxidative stress is important (16). In GEMSP, the presence of vitamins and certain amino acids and their derivatives (alpha-tocopherol-succinate, ascorbic acid, taurine and 5-methoxy-tryptamine) are important neuroprotective components and radical scavengers, exerting a crucial role against nitrosative and oxidative stress $(1,11)$. Cysteine and methionine also act as antioxidants and scavengers contributing to reduced apoptosis and neuronal death induced by reactive oxygen species (17). Alpha-tocopherol and neurotransmitters, such as histamine and 5-methoxy-tryptamine and amino acids, such as histidine act as neuroprotective agents $(1,18)$. Polyunsaturated fatty acids exert a neuroprotective effect and act as free radical scavengers preventing oxidation of cell membrane unsaturated fatty acids $(19,20)$. GEMSP protects and enhances the formation of myelin sheath $(11,14)$. Deficiencies in essential fatty acids impair myelin synthesis (21) and hence it is possible that the fatty acids present in GEMSP are involved in the process of remyelinization, as observed in a chronic EAE model (14). Additionally, it is known that the oleic acid, present in GEMSP, acts as a neurotrophic factor in neurons (22) and that fatty acids exert anti-inflammatory activity (7). 
Previous studies have proposed the involvement of bacteria in the pathogenesis of MS (23-25). Commensal bacteria usually protect the organism, however, may cause autoimmune processes when bacteria pass through the mucosal epithelium to the submucosal tissues. Certain bacterial components act as super-antigens, and induce the proliferation of autoreactive lymphocyte clones and the raising of autoantibodies. In response to lipopolysaccharides (LPS), immune cells produce reactive oxygen species and pro-inflammatory mediators, which are involved in oxidative stress, the demyelination processes and axonal damage (26). Systemic injection of LPS leads to the invasion of the brain by granulocytes and a breakdown of the blood-brain barrier (27). This breakdown may allow the toxins produced by the bacteria to enter the central nervous system. Inside the central nervous system, LPS may exert direct neurotoxic action on microglial cells and LPS is associated with extensive oligodendrocyte death due to LPS-induced neurotoxicity (28). LPS may bind to the blood-brain barrier through their lipid A (gram-negative bacteria) and cross the barrier. Lipid A provides the hydrophobic anchor that secures the molecule within the membrane, while the polysaccharide component interacts with the external environment, including the defences of the host species (29). Thus, an important goal would be to inhibit this induction process by preventing the interaction of bacteria with target cells. In this sense, an amide linkage to fatty acid is preferable to an unstable ester linkage to achieve the same fatty acid presentation as the lipid A component of the LPS of gram-negative bacteria. The similar structure of lipid A and fatty acid, linked to PLL, leads to the neutralization of LPS through the onset of lipid bilayer formation. This approach may prevent LPS from linking to target cells and may allow the inhibition of chronicity factors (6).

In conclusion, preclinical and clinical data suggest that GEMSP presents a novel therapeutic agent/approach for the treatment of MS. GEMSP targets the different aspects of MS rather than the inflammatory aspect alone. GEMSP decreases inflammatory mechanisms, controls oxidative stress, counteracts demyelination, acts as a neuroprotector and fights chronic factors. The present data, using a larger population of MS patients than previous clinical studies $(1,6)$, confirms the beneficial effects of GEMSP that were previously reported.

\section{Acknowledgements}

The present study was supported by the Institut pour le Développement de la Recherche en Pathologie Humaine et Thérapeutique (Talence, France). The authors would like to thank Mr. B. Combes for the statistical analysis (Stalphamis; Le Bourg, France).

\section{References}

1. Mangas A, Coveñas R, Bodet D, Duleu S and Geffard M: A new drug candidate (GEMSP) for multiple sclerosis. Curr Med Chem 16: 3203-3214, 2009.

2. Sawcer S, Hellenthal G, Pirinen M, Spencer CC, Patsopoulos NA, Moutsianas L, Dilthey A, Su Z, Freeman C, Hunt SE, et al; International Multiple Sclerosis Genetics Consortium; Wellcome Trust Case Control Consortium 2: Genetic risk and a primary role for cell-mediated immune mechanisms in multiple sclerosis. Nature 476: 214-219, 2011.
3. Kutzelnigg A, Lucchinetti CF, Stadelmann C, Brück W, Rauschka H, Bergmann M, Schmidbauer M, Parisi JE and Lassmann $\mathrm{H}$ : Cortical demyelination and diffuse white matter injury in multiple sclerosis. Brain 128: 2705-2712, 2005.

4. Villar LM, Sádaba MC, Roldán E, Masjuan J, González-Porqué P, Villarrubia N, Espiño M, García-Trujillo JA, Bootello A and Alvarez-Cermeño JC: Intrathecal synthesis of oligoclonal IgM against myelin lipids predicts an aggressive disease course in MS. J Clin Invest 115: 187-194, 2005.

5. Mangas A, Coveñas R and Geffard M: New drug therapies for multiple sclerosis. Curr Opin Neurol 23: 287-292, 2010.

6. Geffard M, de Bisschop L, Duleu S, Pouns O, Ferran G, Bassede A, Hassaine N, Autran JL, Bodet D, Mangas A and Coveñas R: Endotherapia. Antiinflamm Antiallergy Agents Med Chem 9: 197-211, 2010.

7. Geffard M, Duleu S, Bessede A, Vigier V, Bodet D, Mangas A and Coveñas R: GEMSP: A new therapeutic approach to multiple sclerosis. Cent Nerv Syst Agents Med Chem 12: 173-181, 2012.

8. Shih IL, Van YT and Shen MH: Biomedical applications of chemically and microbiologically synthesized poly(glutamic acid) and poly(lysine). Mini Rev Med Chem 4: 179-188, 2004.

9. Geffard M, de Bisschop L, Duleu S, Hassaine N, Mangas A and Coveñas R: Endotherapia: A new frontier in the treatment of multiple sclerosis and other chronic diseases. Discov Med 10: 443-451, 2010.

10. Mangas A, Coveñas R, Bodet D, Dabadie MP, Glaize G and Geffard M: Evaluation of the effects of a new drug on brain leukocyte infiltration in an experimental model of autoimmune encephalomyelitis. Lett Drug Des Discov 3: 138-148, 2006.

11. Mangas A, Coveñas R, Bodet D, de León M, Duleu S and Geffard M: Evaluation of the effects of a new drug candidate (GEMSP) in a chronic EAE model. Int J Biol Sci 4: 150-160, 2008.

12. Villoslada P: Neuroprotective therapies for multiple sclerosis and other demyelinating diseases Mult Scler Demyel Dis 1: 1, 2016.

13. Mangas A, Coveñas R, Bodet D and Geffard M: Antisera and immunocytochemical techniques. In: Brain molecules: From vitamins to molecules for axonal guidance. Mangas A, Coveñas R and Geffard M (eds). Transworld Research Network, Trivandrum, pp1-25, 2008.

14. Mangas A, Vecino E, David Rodríguez F, Geffard M and Coveñas R: GEMSP exerts a myelin-protecting role in the rat optic nerve. Neurol Res 35: 903-911, 2013.

15. Noseworthy JH, Lucchinetti C, Rodríguez $M$ and Weinshenker BG: Multiple sclerosis. N Engl J Med 343: 938-952, 2000.

16. van Meeteren ME, Teunissen CE, Dijkstra CD and van Tol EA: Antioxidants and polyunsaturated fatty acids in multiple sclerosis. Eur J Clin Nutr 59: 1347-1361, 2005.

17. Agar J and Durham H: Relevance of oxidative injury in the pathogenesis of motor neuron diseases. Amyotroph Lateral Scler Other Motor Neuron Disord 4: 232-242, 2003.

18. Crouzin N, de Jesus Ferreira MC, Cohen-Solal C, Aimar RF, Vignes $\mathrm{M}$ and Guiramand J: Alpha-tocopherol-mediated long-lasting protection against oxidative damage involves an attenuation of calcium entry through TRP-like channels in cultured hippocampal neurons. Free Radic Biol Med 42: 1326-1337, 2007.

19. Blondeau N, Widmann C, Lazdunski M and Heurteaux C: Polyunsaturated fatty acids induce ischemic and epileptic tolerance. Neuroscience 109: 231-241, 2002.

20. Yoshida H, Yanai H, Namiki Y, Fukatsu-Sasaki K, Furutani N and Tada N: Neuroprotective effects of edaravone: A novel free radical scavenger in cerebrovascular injury. CNS Drug Rev 12: 9-20, 2006

21. Di Biase A and Salvati S: Exogenous lipids in myelination and myelination. Kaohsiung J Med Sci 13: 19-29, 1997.

22. Medina JM and Tabernero A: Astrocyte-synthesized oleic acid behaves as a neurotrophic factor for neurons. J Physiol Paris 96: 265-271, 2002

23. Ebringer A, Hughes L, Rashid T and Wilson C: Acinetobacter immune responses in multiple sclerosis: Etiopathogenetic role and its possible use as a diagnostic marker. Arch Neurol 62: 33-36, 2005.

24. Hughes LE, Bonell S, Natt RS, Wilson C, Tiwana H, Ebringer A, Cunningham P, Chamoun V, Thompson EJ, Croker J, et al: Antibody responses to Acinetobacter spp. and Pseudomonas aeruginosa in multiple sclerosis: Prospects for diagnosis using the myelin-acinetobacter-neurofilament antibody index. Clin Diagn Lab Immunol 8: 1181-1188, 2001. 
25. Furrows SJ, Hartley JC, Bell J, Silver N, Losseff N, Stevenson S, Chapman M, Thompson EJ, Ridgway GL and Giovannoni G: Chlamydophila pneumoniae infection of the central nervous system in patients with multiple sclerosis. J Neurol Neurosurg Psychiatry 75: 152-154, 2004.

26. Victor VM and De La Fuente M: Changes in the superoxide production and other macrophage functions could be related to the mortality of mice with endotoxin-induced oxidative stress. Physiol Res 52: 101-110, 2003.

27. Bohatschek M, Werner A and Raivich G: Systemic LPS injection leads to granulocyte influx into normal and injured brain: Effects of ICAM-1 deficiency. Exp Neurol 172: 137-152, 2001.
28. Lehnardt S, Lachance C, Patrizi S, Lefebvre S, Follett PL, Jensen FE, Rosenberg PA, Volpe JJ and Vartanian T: The toll-like receptor TLR4 is necessary for lipopolysaccharide-induced oligodendrocyte injury in the CNS. J Neurosci 22: 2478-2486, 2002.

29. Johnson KP, Brooks BR, Cohen JA, Ford CC, Goldstein J, Lisak RP, Myers LW, Panitch HS, Rose JW, Schiffer RB, et al: Extended use of glatiramer acetate (Copaxone) is well tolerated and maintains its clinical effect on multiple sclerosis relapse rate and degree of disability. Copolymer 1 Multiple Sclerosis Study Group. Neurology 50: 701-708, 1998. 